\title{
OBSERVATIONS OF VENUS AT 8.6-MM WAVELENGTH
}

\author{
J. E. GIBSON AND R. J. MC EWAN \\ Radio Astronomy Branch, US. Naval Research Laboratory \\ Washington, D.C., U.S.A.
}

Mayer, McCullough, and Sloanaker [1] and Drake and Ewen [2] have measured centimeter wavelength radiation from several of the planets; the former at 3.15 and $9.4 \mathrm{~cm}$, the latter at a wide bandwidth centered near $3.75 \mathrm{~cm}$. The first measurements were the observations of Venus by Mayer et al., made near inferior conjunction in late spring 1956. These were obtained with a sufficiently favorable signal-to-noise ratio to permit direct recognition of Venus in individual diurnal-rate scans, and a brightness temperature of $560 \pm 73^{\circ} \mathrm{K}$ (mean error) was deduced for the planet at inferior conjunction, i.e. for the dark side of Venus.

Additional observations of Venus were attempted more recently at $8.6-\mathrm{mm}$ wavelength, near the time of conjunction on 1958 January 28. The objectives were (1) to determine the brightness temperature of Venus at this shorter wavelength; and (2) to investigate techniques of detecting very faint cosmic radiation. Prior efforts with the Naval Research Laboratory 50-foot reflector at $8.6 \mathrm{~mm}$ were unsuccessful because of antenna pointing and focusing uncertainties. The present work, therefore, was carried on with a smaller instrument developed particularly for this wavelength.

The instrument consists of a polar-mounted 10-foot precision paraboloid three feet in focal length, and a rapid-switching receiver of the Dicke type, described previously by Gibson [3]. The receiver noise figure is about $13 \mathrm{db}$, and the rms value of the noise flicker for an integration time constant of one second is roughly two degrees in temperature, referred to the antenna feed. The antenna beamwidth is 0.2 degrees between half-power points, and the electric polarization plane is north-south.

By assuming an antenna aperture efficiency of 60 per cent, it was estimated that the antenna temperature increase for Venus would be two degrees, or one-half of the three-and-a-half degrees observed at $3.15 \mathrm{~cm}$ with the 50 -foot reflector. The expected two-degree deflection for Venus is below the threshold of reliable recognition, if an integration time constant (the time required for the response to a step function to reach $1-1 / e$ of the final value) of three seconds or less is used to insure negligible error at scanning speeds as fast as one-quarter degree per minute with this beamwidth. A slower scanning speed was not available; therefore, the use of long time constants was not attempted. Moreover, the relatively slow fluctuations of background level (owing to atmospheric effects) sometimes seen at this wavelength make it 
inadvisable to rely on long integration time constants to improve the effective sensitivity. Therefore, it was planned to obtain the necessary additional integration by detailed averaging of a group of scan recordings.

Observations were undertaken on four occasions in late January and early February. On the first period of observation (January 29), the recordings were made at the diurnal scanning rate (one-quarter degree per minute) with an integration time constant of three seconds. The peak-to-peak value of the flicker was about eight degrees; elimination of the enhanced low-frequency components having a period greater than 30 seconds would reduce the peakto-peak flicker to almost three degrees. More recent tests have shown that the excessive low-frequency content of the flicker is not inherent when the receiver is operating correctly; thus, the potential system sensitivity is greater than that attained during these observations of Venus. As previously mentioned, some, if not all, of this deterioration seems to be due to atmospheric self-emission fluctuations.

Other observations (February 5,6, and 11) were carried out by motor-driven scanning in declination at a rate of about one degree per minute, with the antenna tracking Venus in hour angle. A one-degree arc was scanned in declination, which was swept alternately by automatic switches in the antenna controls. The integration time constant was one second. The purpose of these faster scans was to obtain better contrast between the deflection due to Venus and the slowly undulating background. Superficial consideration of the results indicates doubt that source recognition is improved, but the greater number

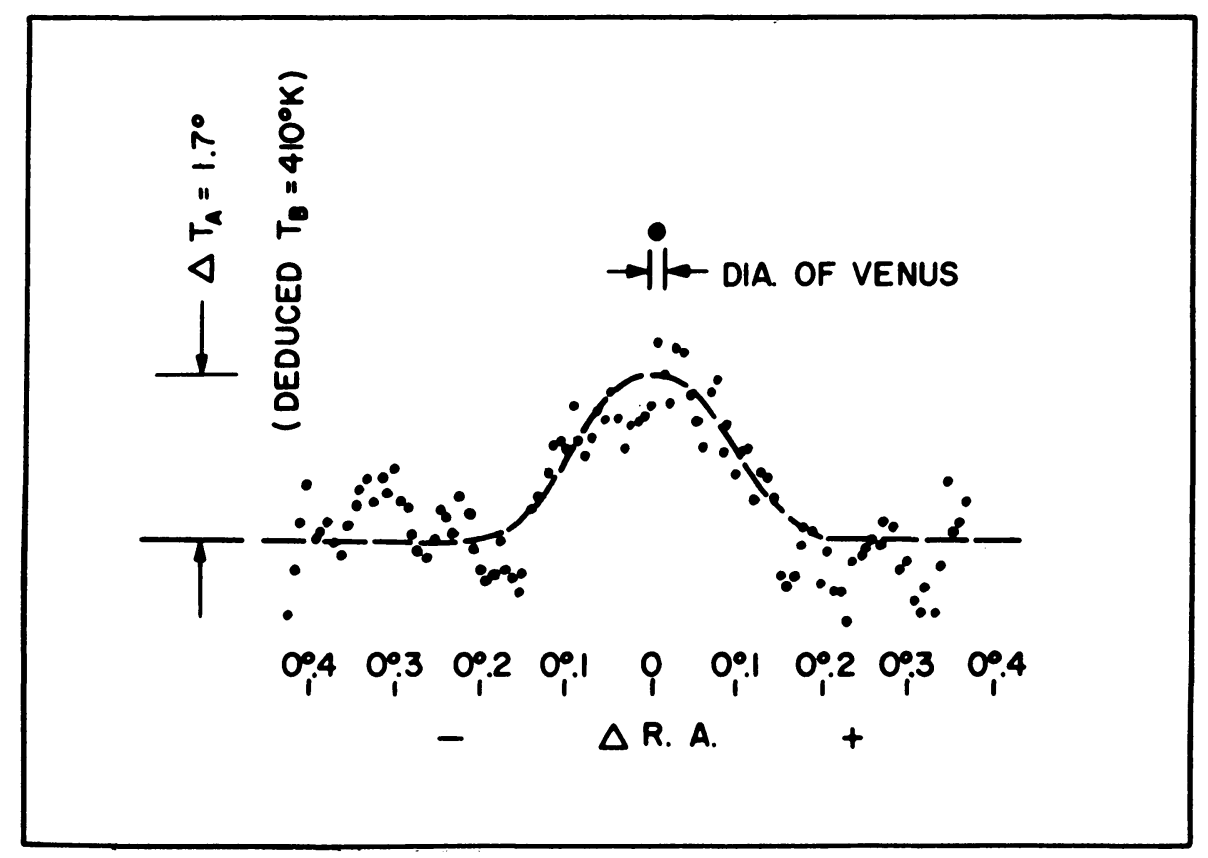

FIG. 1. The deflection derived from averaging fifteen scans of Venus. 
of scans obtained in a given period has statistical value when suitable observing time is limited.

The west-to-east scan recordings of January 29 have been averaged; they yield the scatter of points shown in Fig. 1. Each point is the average of the instantaneous deflections of 15 recordings read at the particular right-ascension increment relative to Venus. The mean deflection represented by the points is indicated by the dashed line, the base level of which is the average of the points lying outside the full width of the antenna beam. The apparent discrepancy between the averages of the points east and west of Venus is attributed to the random flicker in the recordings, rather than to any actual variation in background level, since there was no progressive variation in gain or background reference level during the observation period.

The mean deflection at the position of Venus was found to correspond to an increase in antenna temperature of about 1.7 degrees, including a correction for atmospheric attenuation. For a source size of 62 seconds of arc in diameter, i.e. a solid angle of $71 \times 10^{-9}$ steradians, and with the antenna efficiency assumed to be 60 per cent, this antenna temperature corresponds to a brightness temperature for Venus of about $410^{\circ} \mathrm{K}$. The accuracy of this value is limited chiefly by the flicker in the recordings and by the uncertainty in the assumed antenna efficiency. These uncertainties, taken together with the lesser errors of calibration and antenna pointing, were estimated to amount to a probable error of roughly 40 per cent. Thus, the brightness temperature of the dark side of Venus is probably between 250 and $570^{\circ} \mathrm{K}$ at this wavelength.

Grateful acknowledgments are due C. H. Mayer and R. M. Sloanaker for valuable discussions of the techniques and interpretation of their observations, and to F. L. Hennessey of the Microwave Antennas Branch of NRL for estimates of antenna characteristics.

\section{REFERENCES}

[1] Mayer, C. H., McCullough, T. P., and Sloanaker, R. M. Ap. J. 127, 1, 11, 1958.

[2] Drake, F. D., and Ewen, H. I. Proc. I.R.E. 46, 53, 1958.

[3] Gibson, J. E. U.S. Nav. Res. Lab. Report 4864, December 1956. 